\title{
Estimation of azimuth of a macro cell through user data for LTE access network
}

\author{
BRIJESH SHAH ${ }^{1, *}$, GAURAV DALWADI ${ }^{1}$, RAHUL BHASKER $^{1}$, HARDIP SHAH $^{2}$ and \\ NIKHIL KOTHARI ${ }^{2}$ \\ ${ }^{1}$ Reliance Jio Infocomm Ltd, Navi Mumbai 400701, India \\ ${ }^{2}$ Dharmsinh Desai University, Nadiad 387001, India \\ e-mail: brijesh_i_shah@yahoo.com; dalwadigaurav@gmail.com; rahulbhasker84@gmail.com; \\ echardip@ddu.ac.in; nil_kothari@ddu.ac.in
}

MS received 1 February 2019; revised 31 March 2019; accepted 12 May 2019; published online 17 June 2019

\begin{abstract}
The number of antennas on a site increases due to a simultaneous deployment of multi-band and multi-mode radios to combat extremely growing data demand in the network. The correct values of physical parameters of antennas, including azimuth, height and tilt, are essential to optimize the radio frequency (RF) network automatically. It is seen that poor results in RF network optimization are mainly due to incorrect azimuth. The proposed algorithm can estimate the azimuth of an antenna in the field using passive monitoring data from the user equipment. It has been developed to identify the correct value of azimuth without doing the field audit, which can significantly reduce the time for optimization and operational expenditure (OPEX) as well. The field trial reveals that the estimated azimuth value closely matches within $\pm 12^{\circ}$ range in comparison to the actual value in the field. Moreover, field results show that the same algorithm is equally applicable for urban and rural morphologies as well. It can also be automated to sanctify the physical site database with proper azimuth values at large level without introducing any kind of human error.
\end{abstract}

Keywords. Antenna azimuth; LTE access network; network optimization; passive monitoring data; reference signal received power (RSRP); user equipment (UE).

\section{Introduction}

An antenna is an essential element in the cellular network as it decides the footprint of the radio frequency (RF) coverage. Conventionally three sectors are used at the site location in 2G, 3G and 4G telecommunication networks, which lead to the requirements of three times sector antenna compared with the number of sites in the network $[1,2]$. Because of extensive growth in mobile data requirement since the last few years, operators are forced to use multi-band and multi-technology sector antennas at the same site location [3-5]. Planning of antenna parameters such as azimuth and tilt becomes very important as it decides the RF coverage and signal quality at a user equipment (UE), which in turn decides the overall capacity of the network. It is essential that the antennas installed in the field should have the planned value of azimuth and tilt in order to have enough coverage in the designated area. If the antennas are not installed as per the planning input, there could be a large number of either coverage holes or intercell interference in the network. Further, optimization

*For correspondence cannot provide the desired results in such cases due to incorrect input of antenna parameters. Hence, the correct value of antenna parameters in the centralized database is mandatory to achieve the best outcome from the RF optimization in the network.

With the advancement of the antenna technologies, the antenna tilt change is done remotely using remote electrical tilt (RET) feature through a centralized server that ensures the correct value in the database as per the actual value in the field. However, the antenna azimuth adjustment still requires a field visit and manual entry of the new value in the database. Manual entry is an erroneous process and may not guarantee the correct value of azimuth in the database for all sectors in the network. Hence the correct value of azimuth as per the field data is a major problem for any cellular operator. As on date, operators rely on the field person discipline and the workmanship and believe that azimuth measurement carried by field person is always correct and entered value in the database is also correct. There is no mechanism to validate azimuth correctness in the database in comparison with the actual value in the field. Moreover, changes in these antenna parameters are quite often in the network because of various activities such as RF optimization, network upgradation, resolution of customer 
complaints, etc., which further add complexity in maintaining the correct azimuth database. In order to ensure the correct database as per actual value in the field, network operators have to initiate field visit on a regular basis and correct all such mismatches. This is a herculean task, which impacts operating cost (OPEX) and requires huge manpower. This issue becomes more severe in LTE-based 4G network, since the number of sites is too many compared with GSM and CDMA network in order to provide good RF coverage and better user experience [6-10].

Nowadays, many of the LTE operators use antennas with RET, which helps in maintaining the correct value of electrical tilt in the database [11]. However, the correct value of azimuth is still a big problem for all kinds of cellular network and will be equally valid for the $5 \mathrm{G}$ network as well. There is an evolution in the measurement approach for antenna azimuth in the field. Azimuth measurement has evolved from the conventional practice of using a magnetic compass to recently commercially available antenna alignment tool (AAT) [12]. However, these tools require field visit and manpower on a regular basis to have a periodic measurement. In addition, it is a time-consuming process and becomes a bottleneck for the activities related to RF optimization. Of late, there are new approaches such as machine learning, block chains and big data analytics, which are the potential technologies to automate the RF network on continuous basis; however, the performance of these techniques ultimately depends on the accuracy of antenna azimuth. To overcome the above challenges, we have proposed a novel algorithm to estimate azimuth based on UE data, which are collected through passive monitoring. This state of the art algorithm also greatly reduces inaccuracy caused by instrument, field auditor and data recorder. An automation of this algorithm will significantly reduce the efforts of field audit to have the correct azimuth database. We have validated our proposed algorithm for LTE network in Lucknow city of India for urban morphologies and also applied to a village locality of Kerala state to verify its performance in rural clutter. The estimated azimuth data have been found quite matching with field audit results with a good degree of accuracy.

The rest of the paper is structured as follows. Section 2 describes various prevalent methods of azimuth measurement. The proposed algorithm using UE data is elaborated in section 3. A case study of azimuth estimation in LTE network of Lucknow city has been discussed in section 4 . Finally, in section 5, we conclude the paper and recommend the future research scope on this topic.

\section{Various prevalent methods for azimuth measurement}

Currently, there are various methods prevalent in practice for azimuth measurement. In this section, these methods with their pros and cons have been briefly described.

\subsection{Measurement using the magnetic compass}

This is a conventional approach mostly used in the field by many network operators in which the field engineer measures the azimuth by aligning the magnetic compass in the direction of the antenna facing and then records the direction of the magnetic needle to capture the azimuth value. This method is prone to faulty measurement due to the parallax error. Also, the magnetic compass needle may get deflected because of a metal structure nearby the antenna or due to manual intervention during recording the value [13]. The azimuth error could be more than $\pm 20^{\circ}$ even by using this technique. Hence, this method is not the correct approach.

\subsection{Measurement using AAT}

This method using the AAT gives the correct value of antenna azimuth, which is error free since it does not involve any human intervention. AAT utilizes differential GPS technology to measure the azimuth and the tool automatically records all alignment parameters, including azimuth, along with latitude, longitude and height of the antenna above the ground [14]. These records can be automatically updated to the network database. This method is getting more popular. However, the cost of AAT is too high and the measurement process is a little complex in comparison with the magnetic compass. Also, another limitation is that whenever we need to collect the antenna azimuth measurement, it has to be physically attached to the antenna every time. This causes a time-consuming affair during measurement.

\subsection{Antenna azimuth system (AAS)}

Antenna manufacturing vendors have come up with a solution in which antenna azimuth system (AAS) can be connected to the antenna and any azimuth change can be captured and updated in the database through operations and maintenance centre of the operator [15]. This also involves huge cost and extra devices and its mounting requirement and hence this solution is not viable for all the sectors in the network.

As the network expands with more sites and multiple spectra, the number of antennas installed in the network will also increase considerably. There may also be frequent changes happening in the network as a part of the optimization and maintenance, which make daily tracking hard stuff. It will be difficult to ensure that the antennas installed on the sites are as per the planned value and also there may be cases where the azimuth might have been changed on the site physically but the site database may not be updated due to the negligence of field staff. Therefore, estimating the azimuth based on the UE data will be able to provide the correctness of azimuth database. The proposed 


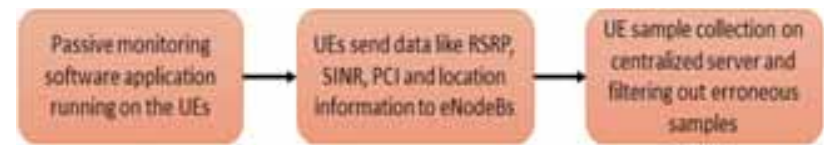

Figure 1. Flow chart of UE data collection.

algorithm for azimuth estimation is discussed in the next section.

\section{Proposed azimuth estimation method}

To address the afore-mentioned teething issues related to azimuth database, a novel algorithm has been proposed in this section, which helps estimating the azimuth of the antenna installed on the site with the help of UE data. The UE passive monitoring data carry information like latitude, longitude of user location, physical cell identity (PCI) of the serving cell and reference signal received power (RSRP) received at UE from the serving cell $[5,16,17]$.

Figure 1 depicts the process for collecting the UE samples through a passive monitoring software application. This application automatically captures the samples periodically in the background without any user-specific intervention. UE stores the samples for the entire day from various events such as charging trigger, RSRP limit crossover during poor coverage, handover occurrence, low battery alarm, etc. and uploads to the centralized server once in a day through LTE interface. Each UE sample includes the information of RSRP, signal to interference and noise ratio (SINR), PCI and latitude/longitude of UE location [18-20]. UE samples are processed through various filtering steps before using as an input for our proposed azimuth estimation algorithm. These filtering steps act on removal of erroneous samples such as samples without

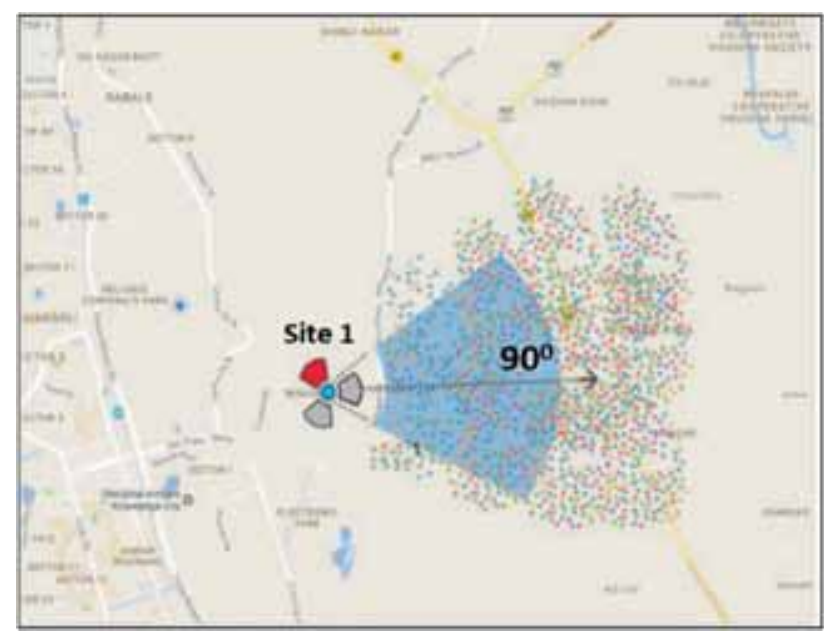

Figure 2. Example of UE samples collection for the same PCI. location information, samples with poor GPS accuracy and values of RSRP and SINR beyond specified range, samples collected by known low-quality UEs, samples with no PCI information, etc. Figure 2 illustrates one example of the distribution of UE samples with the same PCI for one sector. Most of the samples lie in one cylindrical cone as per the expectation. The angular width of the cone depends on the antenna beamwidth.

The detailed workflow for the proposed algorithm is explained in figure 3. The algorithm follows the bottom-up approach and estimates the azimuth based on the angular region where maximum UE samples are available for the corresponding cell. The aperture of the angular region is the same as antenna beamwidth for the corresponding cell. The accuracy of the proposed algorithm depends on the number of UE samples available for the corresponding cell. As the numbers of UE samples are more, the azimuth estimation would be more accurate. Therefore, a minimum threshold has been kept for the number of UE samples to estimate the azimuth. In our simulation, we have kept a threshold of $100 \mathrm{UE}$ samples for a particular cell. The step-by-step details of the proposed algorithm are given as follows:

a) Create a cone whose aperture $\left(A_{p}\right)$ is as per the antenna beam width.

b) Move the cone in $1^{\circ}$ step for the entire $360^{\circ}$ with respect to true north as shown in figure 4 .

c) Calculate the number of samples for each cone.

d) If any cone has more than $60 \%$ of samples for the corresponding PCI, identify the cone with a maximum number of samples. The cone angular number $\left(N_{x}\right)$ is with respect to true north. For this case, estimated azimuth $\left(A_{E}\right)$ is given as

$$
A_{E}=N_{x}+\left(A_{p} / 2\right) .
$$

e) If there is no cone with more than $60 \%$ samples in $360^{\circ}$ angles, use the logic of 'cone aperture expansion' as shown in figure $5(\mathrm{a})$. Increase the width of the cone in $1^{\circ}$ step and repeat the process for identifying a cone with the maximum number of samples as a part of cone aperture expansion logic. This step is to be repeated until cone aperture reaches up to $90^{\circ}$. Here, cone expansion limitation $\left(90^{\circ}\right)$ is decided on the basis of first side lobe level of the antenna radiation pattern. This may vary as per the position of first side lobe in the radiation pattern.

f) Calculate a centroid using all the samples in a cone where maximum samples are found. The angle between true north and centre axis of the centroid is considered as an estimated azimuth.

Figure 5(b) shows the calculation of centroid of random UE samples. For example, $x_{1}, x_{2}, x_{3}, \ldots, x_{n}$ are the $x$-coordinates of UE samples and $y_{1}, y_{2}, y_{3}, \ldots, y_{n}$ are the $y$-coordinates of UE samples with respect to site location as an origin. The centroid of such $n$ samples is given by 


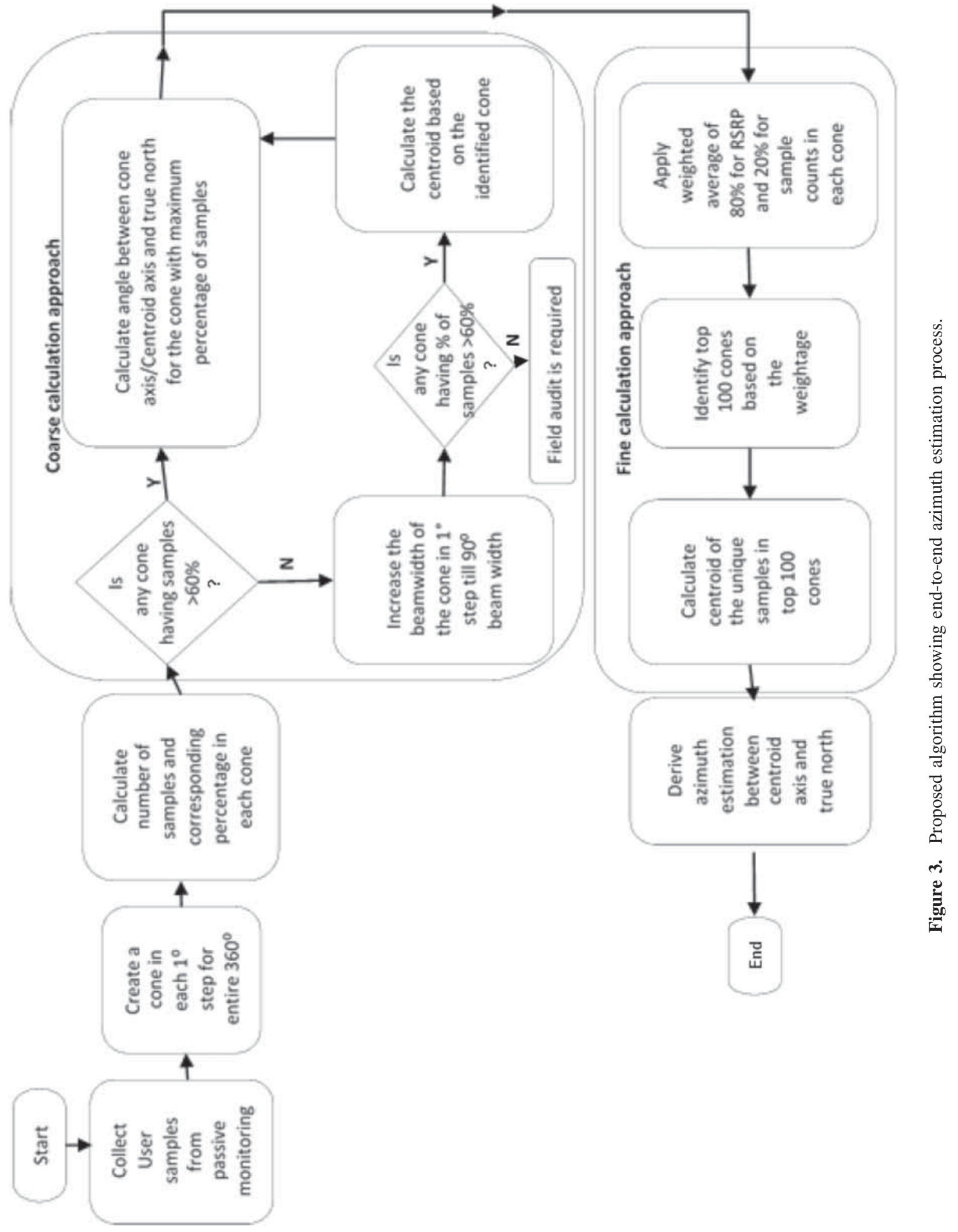




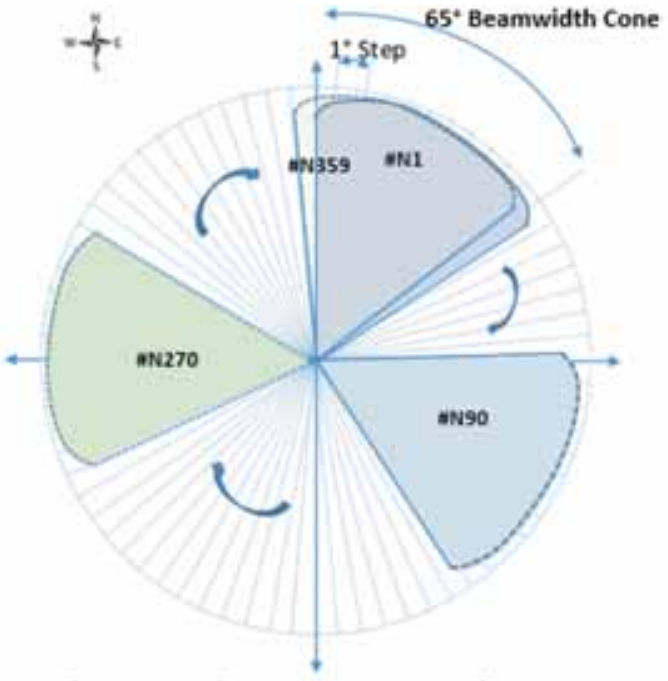

- Rotate $65^{\circ}$ Cone in $360^{\circ}$ (w.r.t True North) in $1^{\circ}$ step

- $\#$ N1, \#N2, \#N.... \#N359-Number of cones created in $1^{\circ}$ Step up to $360^{\circ}$

Figure 4. Creation of cone and clockwise rotation.

$$
\begin{gathered}
(<x>,<y>)=\left(\frac{\sum_{i}^{n} x_{i}}{n}, \frac{\sum_{i}^{n} y_{i}}{n}\right), \\
\theta=\left|\arctan \left(\frac{<y>}{<x>}\right)\right| .
\end{gathered}
$$

Now, the estimated azimuth $\left(A_{E}\right)$ in degree with respect to true north is given by

$$
\begin{array}{r}
A_{E}=90-\theta \quad \text { if centroid falls in } 1^{\text {st }} \text { quadrant } \\
A_{E}=270+\theta \quad \text { if centroid falls in } 2^{\text {nd }} \text { quadrant }, \\
A_{E}=270-\theta \quad \text { if centroid falls in } 3^{\text {rd }} \text { quadrant } \\
A_{E}=90+\theta \text { if centroid falls in } 4^{\text {th }} \text { quadrant }
\end{array}
$$

Here, steps (a)-(f) show the azimuth estimation approach by coarse calculation.

g) For fine-tuning of the azimuth estimation, we have performed further deep analysis using weighted average approach. The users at the boresight of the antenna have better RSRP and number of the samples in this region is also higher. Sometimes, due to hindrances and obstruction caused by buildings, trees, etc., UE samples are diverted and fall in non-line-of-sight locations due to reflection, refraction and scattering effect, which is a common phenomenon especially for dense or medium urban scenarios. In such cases, only concentration of samples does not provide correct estimation, but signal strength also plays a pivotal role compared with the sample count. Hence, an inclusion of proper weightage to average RSRP along with average sample concentration in coarse calculation-based approach can lead to

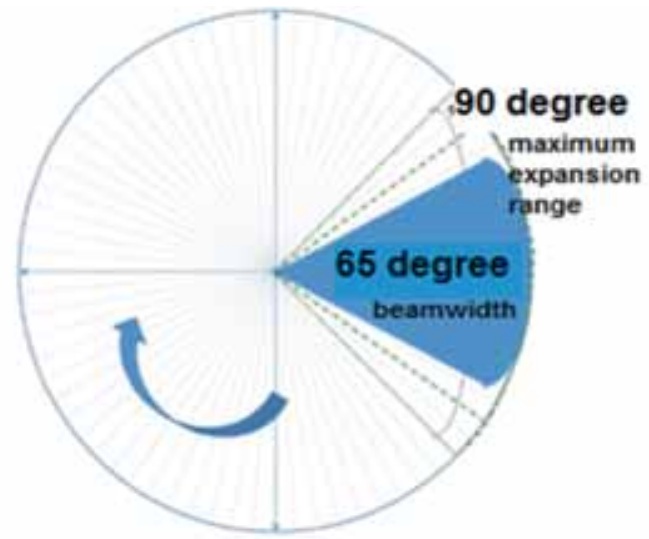

(a)

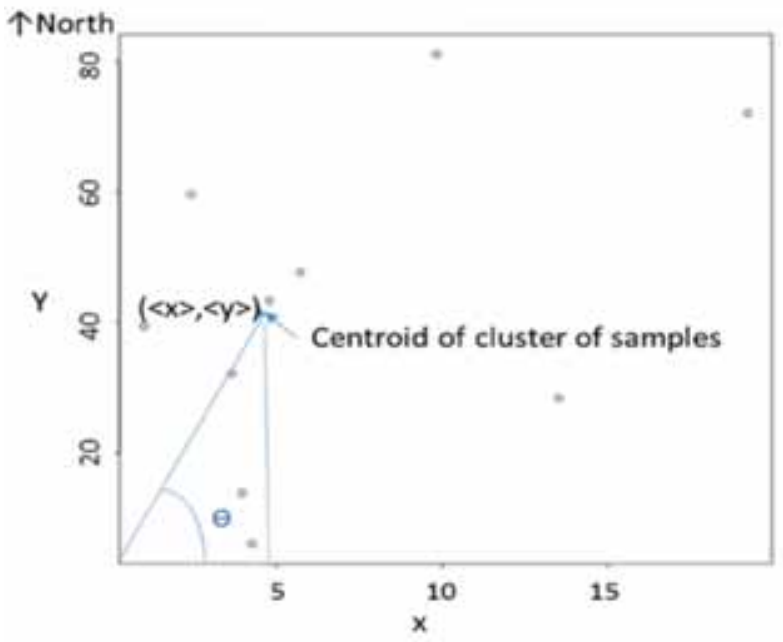

(b)

Figure 5. (a) Cone expansion method in case of UE samples < $60 \%$ and (b) calculation of centroid of random cluster of samples.

higher estimation accuracy. This fine-calculation-based approach is described mathematically in (5) using statistical analysis. Whichever cone has higher $w_{x}$ is considered as the estimated azimuth.

$$
w_{x}=\alpha \cdot\left(\frac{10 \cdot \operatorname{LOG}\left(\frac{\sum_{i}^{n} R S R P_{i}}{10 n}\right)}{R S R P_{\max }}\right)+\beta \cdot\left(\frac{n}{M}\right)
$$

where $\alpha$ is weighting factor for RSRP, $\beta=(1-\alpha)$ is weighting factor for UE sample count, $R S R P_{\max }$ is the maximum average RSRP across all cones, $n$ is total number of samples in $x$ cone and $M$ is maximum number of samples.

The value of $\alpha$ is defined based on the type of clutter and should be either higher than or nearly the same as $\beta$. This is because received signal strength at the UE on the centre axis of the main lobe of an antenna is higher, which 


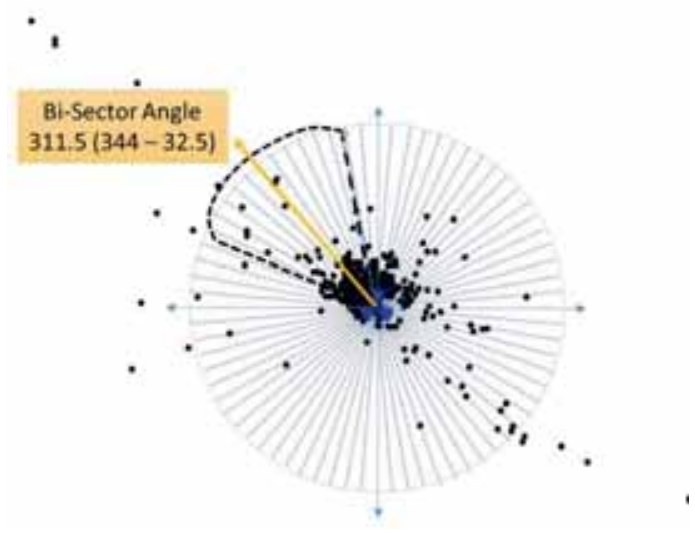

(a)

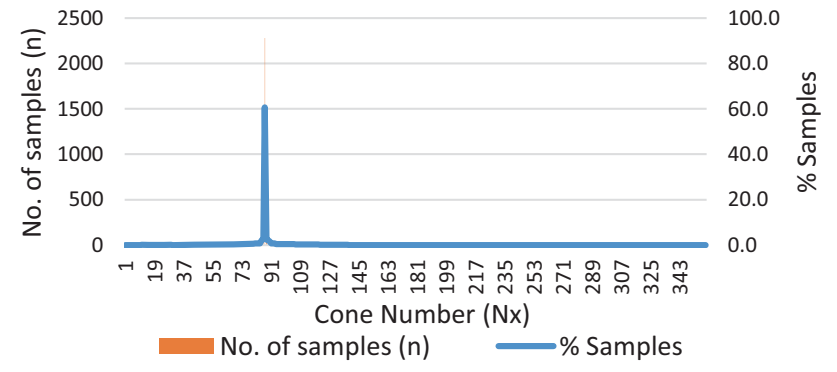

(b)

Figure 6. (a) Case study of Lucknow city - a case of azimuth estimation using maximum number of samples and (b) sample distribution against each cone from $N_{0}$ to $N_{359}$.

increases the RSRP level of UE samples in that area. Therefore, the higher the RSRP value, the greater the probability of azimuth direction. For cells that face towards dense or medium urban areas, the value of $\alpha$ estimated is $[0.6,0.8]$ and for suburban and rural areas the value of $\alpha$ within $[0.4,0.6]$ has been considered in this analysis. The range of $\alpha$ is estimated after practical iterations of multiple sectors from various morphologies and comparative analysis with field data.

Furthermore, the benefit of giving a high weightage to average RSRP parameter over sample count will also reduce the effect of bad samples collected by fast moving UEs since their RSRP value deteriorates significantly compared with stationary UE samples due to high mobility and Doppler effect. In addition to this, UE samples through passive monitoring are collected during network busy hours, probably 8:00 PM-12:00 PM, which is almost 50 times higher than collected during non-busy hours. During this period, stationary UEs are larger than fast moving UEs since more UEs are at residential locations, which further reduce relative sample count for high-speed UEs. Therefore, their overall impact becomes negligible as per (5) in urban clutter types. For rural areas, such sample counts are very low, which eventually nullify their impact according to $(5)$. (h) Identify top 100 cones based on the final combined weightage of RSRP and number of samples in each cone as defined in (5).

(i) Calculate azimuth based on the centroid of the unique samples in top 100 cones by following the same Eqs. (2)-(4).

\section{Case study and field validation}

We have implemented the proposed algorithm for azimuth estimation in MATLAB and validated with the physical database of Lucknow city. Let us estimate azimuth using both approaches.

\subsection{Estimation approach based on coarse calculation}

Figure 6(a) illustrates one example based on the actual user data from the field for azimuth estimation using maximum samples. There are more than 3000 samples collected for the same PCI across $360^{\circ}$ angular span. Percentage of samples distribution is shown in figure 6(b). As can be seen from the figure, only one cone $N_{86}$ carries more than $60 \%$ samples within its aperture of $65^{\circ}$. From (1), we can estimate the azimuth, which is $118^{\circ}$, since it contains maximum of $60.6 \%$ of overall samples. The same sector was measured for azimuth using AAT and as per the field results the azimuth estimated exactly matched the field value.

\subsection{Estimation based on fine calculation}

Azimuth estimation using the weighted average of RSRP value and sample count together will provide a more accurate estimation compared with previously discussed method based on maximum sample count. Table 1 illustrates the weighted average method, where sample count and average RSRP value of the samples inside the cone $N_{x}$ have been tabulated. Here $x$ can vary from $1^{\circ}$ to $365^{\circ}$. We have assumed the value of $\alpha$ factor and $\beta$ factor as 0.8 and 0.2 , respectively, due to dense/medium urban clutter of cell-facing areas.

\subsection{Estimation using both approaches}

Figure 7 shows one example where the azimuth was estimated using coarse calculation or 'based on the maximum number of samples only' as well as the fine calculation using 'weighted average calculation of RSRP and sample count'. It is seen that the azimuth derived from maximum sample count points towards $38^{\circ}$, whereas azimuth estimated using the weightage factor is $347^{\circ}$. During field audit, azimuth measured using AAT was $350^{\circ}$. As per the 
Table 1. A case of azimuth estimation using weighted average of RSRP value and sample count.

\begin{tabular}{|c|c|c|c|c|c|c|c|c|}
\hline $\begin{array}{l}\text { Cone } \\
\text { angle }\end{array}$ & $\begin{array}{c}\text { Sample } \\
\text { count } \\
\text { inside } \\
\text { cone }\end{array}$ & $\begin{array}{l}\text { Average } \\
\text { RSRP } \\
\text { inside } \\
\text { cone } \\
(\mathrm{dBm})\end{array}$ & $\log (10 \log (C / 10)$ & $\begin{array}{c}\text { Normalized } \\
\text { RSRP }\end{array}$ & $\begin{array}{c}\text { Normalized } \\
\text { samples }\end{array}$ & $\begin{array}{c}\text { RSRP } \\
\text { weightage } \\
\text { factor } \\
(\text { average } \\
\text { RSRP } \times 0.8)\end{array}$ & $\begin{array}{c}\text { Sample } \\
\text { weightage } \\
\text { factor }(\text { sample } \\
\text { count } \times 0.2)\end{array}$ & $\begin{array}{c}\text { Final } \\
\text { weightage } \\
(G+H)\end{array}$ \\
\hline 1 & 1489 & -93.29 & $4.68347 \mathrm{E}-10$ & 1.00 & 0.93 & 0.80 & 0.19 & 0.98 \\
\hline 2 & 1463 & -93.29 & 4.69253E-10 & 1.00 & 0.91 & 0.80 & 0.18 & 0.98 \\
\hline 3 & 1538 & -93.41 & $4.55736 \mathrm{E}-10$ & 0.97 & 0.96 & 0.78 & 0.19 & 0.97 \\
\hline 4 & 1544 & -93.49 & $4.47277 \mathrm{E}-10$ & 0.95 & 0.97 & 0.76 & 0.19 & 0.96 \\
\hline 5 & 1546 & -93.52 & 4.44404E-10 & 0.95 & 0.97 & 0.76 & 0.19 & 0.95 \\
\hline 6 & 1554 & -93.59 & 4.37414E-10 & 0.93 & 0.97 & 0.75 & 0.19 & 0.94 \\
\hline 7 & 1561 & -93.66 & $4.3043 \mathrm{E}-10$ & 0.92 & 0.98 & 0.73 & 0.20 & 0.93 \\
\hline 8 & 1484 & -93.62 & $4.34662 \mathrm{E}-10$ & 0.93 & 0.93 & 0.74 & 0.19 & 0.93 \\
\hline 9 & 1551 & -93.71 & $4.26085 \mathrm{E}-10$ & 0.91 & 0.97 & 0.73 & 0.19 & 0.92 \\
\hline 10 & 1565 & -93.73 & $4.23435 \mathrm{E}-10$ & 0.90 & 0.98 & 0.72 & 0.20 & 0.92 \\
\hline 11 & 1563 & -93.73 & $4.23409 \mathrm{E}-10$ & 0.90 & 0.98 & 0.72 & 0.20 & 0.92 \\
\hline . & . & . & . & . & . & . & . & . \\
\hline . & . & . & . & . & . & . & . & . \\
\hline$\cdot$ & . & . & . & . & . & . & . & . \\
\hline . & . & . & . & . & . & . & . & . \\
\hline . & . & . & $\cdot$ & . & . & . & . & . \\
\hline . & . & $\cdot$ & . & . & . & . & . & . \\
\hline 359 & 19 & -106.39 & $2.29464 \mathrm{E}-11$ & 0.05 & 0.01 & 0.04 & 0.00 & 0.04 \\
\hline 360 & 16 & -106.41 & $2.28608 \mathrm{E}-11$ & 0.05 & 0.01 & 0.04 & 0.00 & 0.04 \\
\hline
\end{tabular}

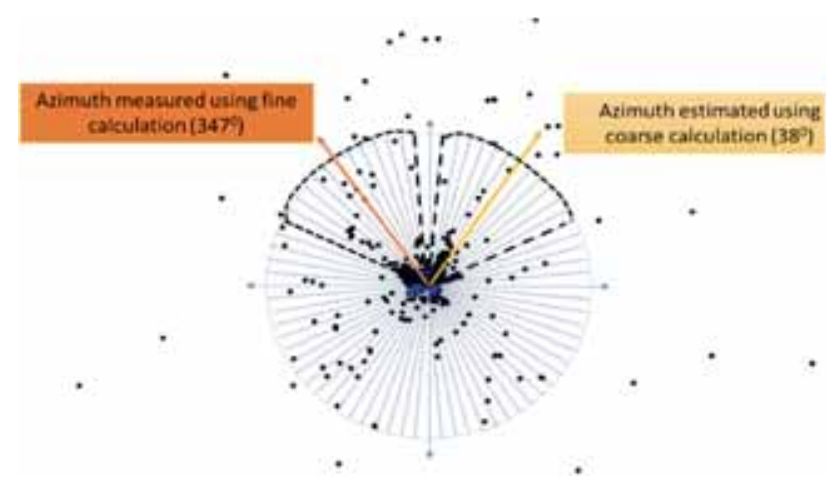

Figure 7. A field validation of a case with coarse calculation and fine calculation methods. field results, the azimuth estimated using the weightage factor exactly matches with the field value.

Figure 8 shows the azimuth estimation using coarse and fine calculation methods and AAT-measured values across 50 LTE macro cells of Lucknow city covering all kinds of morphologies. Table 2 shows the comparative analysis of the field-measured azimuth values using AAT and estimated values based on coarse calculation (using steps (a)(f)) and based on fine calculation (using steps (g)-(i)) for the same LTE macro cells. The estimated azimuth average value with fine calculation converges to field-measured values with an estimation error of $\sim 12^{\circ}$ only compared with coarse calculation, where an estimation error is $\sim 38^{\circ}$.

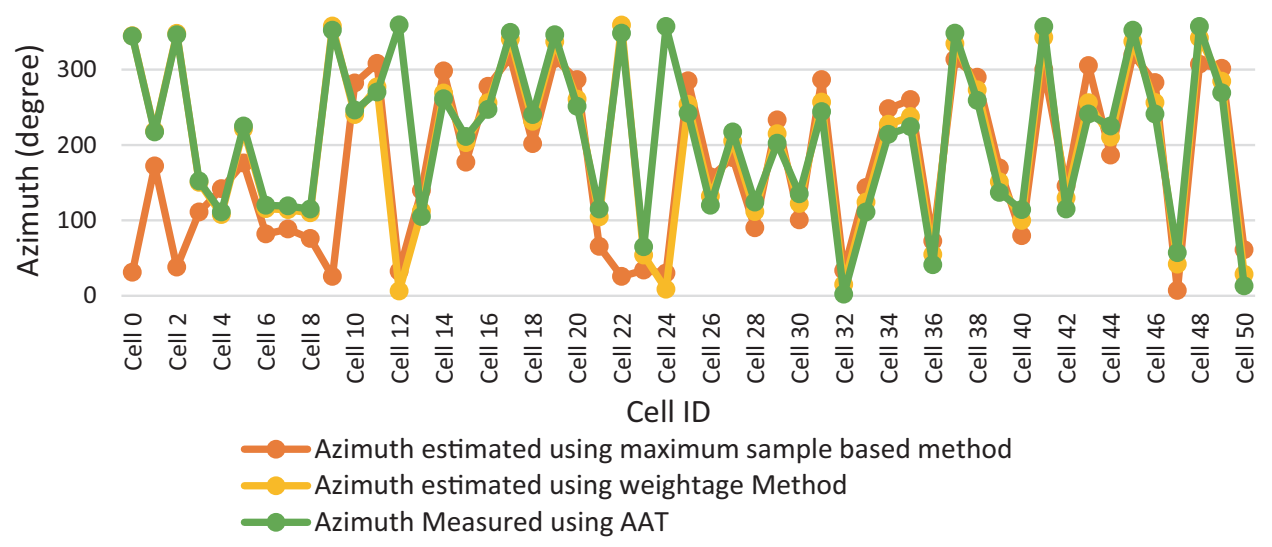

Figure 8. Azimuth estimation using coarse and fine calculation methods and AAT-measured values for Lucknow city. 
Table 2. A summary of average azimuth measured versus estimated values for Lucknow city.

\begin{tabular}{lccc}
\hline Parameter & $\begin{array}{c}\text { Azimuth measured using } \\
\text { AAT (deg) }\end{array}$ & $\begin{array}{c}\text { Estimated azimuth with coarse } \\
\text { calculation (deg) }\end{array}$ & $\begin{array}{c}\text { Estimated azimuth with fine } \\
\text { calculation (deg) }\end{array}$ \\
\hline $\begin{array}{l}\text { Average azimuth of } 50 \\
\begin{array}{l}\text { cells } \\
\text { Estimation error }\end{array}\end{array}$ & 212.4 & 174.2 & 200.4 \\
\hline
\end{tabular}

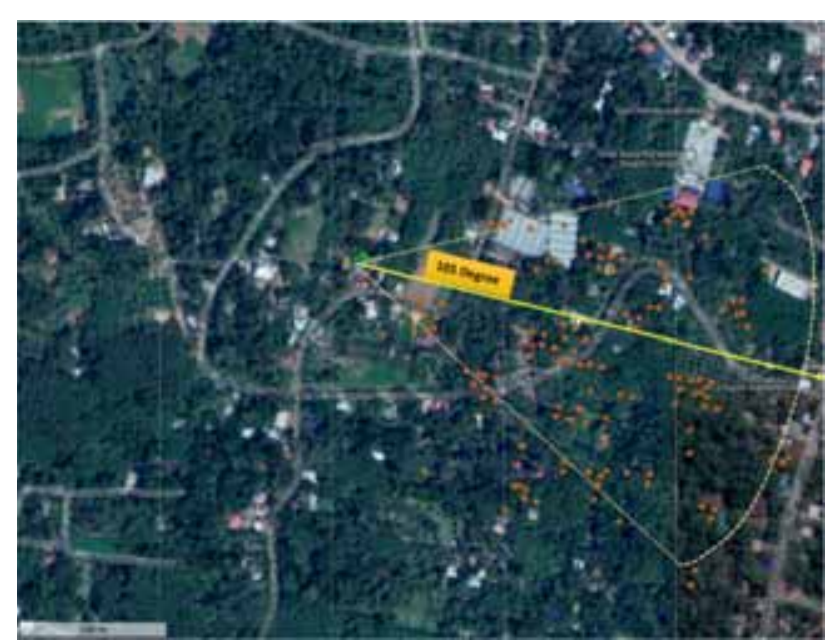

Figure 9. A case study of an azimuth estimation in rural areas.

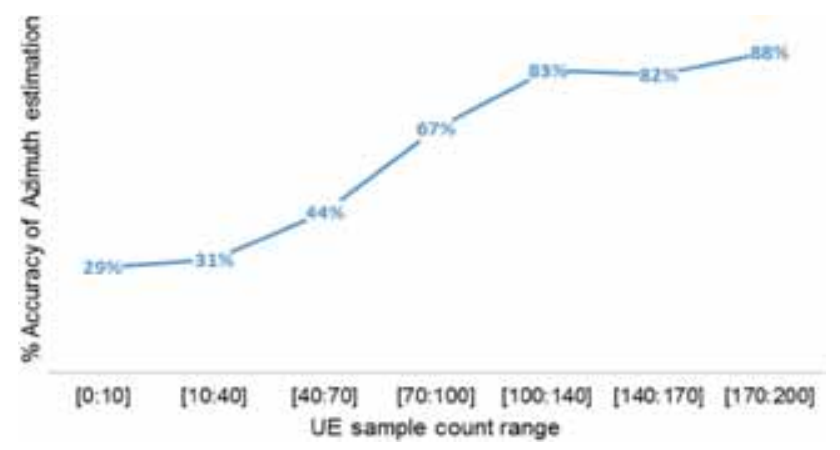

Figure 10. Accuracy of azimuth estimation versus UE samples.

In addition to the urban scenario, one more case study of a rural area is shown in figure 9, where residential areas are scattered and UE samples retrieved are sparse. An azimuth reported in a database for one sector in a village located in Kerala was $150^{\circ}$. While the estimated azimuth using our fine-calculation-based approach identified $105^{\circ}$ and in field visit, it has been observed to be $100^{\circ}$ using AAT tool. It means our fine-calculation-based approach is applicable to any kind of morphologies if enough UE samples are collected.

Furthermore, the limitation of this algorithm is that it works with more than $80 \%$ accuracy if the UE samples (after filtering process mentioned in section 3) are more than 100. This threshold has been derived after multiple iterations and comparative analysis with field data. Such a detailed analysis is captured in figure 10, which shows the percentage accuracy of azimuth estimation against various UE sample counts.

\section{Conclusion}

Due to the evolution of multiband and multi-mode radios for $4 \mathrm{G}$ and upcoming $5 \mathrm{G}$ technologies, the number of antennas on a site location increases. Furthermore, combating with on-going data capacity requirement, sectorization of a site also increases, which leads to upgradation of existing site location with more number of antennas. In such cases, for online or offline optimization, the correctness of antenna azimuth in the database becomes an essential requirement. To avoid conventional field audit and manual recording for azimuth data, here we have proposed a novel algorithm to estimate the azimuth of the antennas using UE trace data collected through passive monitoring. This estimation will help the cellular operators to perform their day to day optimization activities through which they can maintain a healthy network. The algorithm validation also suggests that azimuth estimation method using the weighted average of RSRP value and sample count is more precise and closer to real-time field value compared with coarse calculation approach based on maximum samples. The limitation of this algorithm is that it requires minimum scalable samples $(>100)$ for each cell to proceed with the proposed approach. The case study of 50 macro cells of Lucknow city shows the estimation accuracy within $\pm 12^{\circ}$ compared with the field-measured azimuth value using AAT, which further raises the confidence of estimation algorithm using fine calculation approach. Furthermore, field results show that the same algorithm is equally valid for urban and rural morphologies as well. Also, it can be automated to sanctify the physical site database with proper azimuth values at large level without introducing any kind of human error.

\section{References}

[1] Rappaport T 2009 Wireless communications-principles and practice, 2nd ed. Noida, India: Pearson Education 
[2] Balanis C A 2016 Antenna theory: analysis and design, 4th ed. Toronto, Canada: Wiley Publication

[3] Ngo H Q, Larsson E G and Marzetta T L 2013 Energy and spectral efficiency of very large multiuser MIMO systems. IEEE Trans. Commun. 61: 1436-1449

[4] Zheng Z, Wang J, Peng X and Zhao C 2014 Coexistence analysis between WCDMA and TD-LTE systems in multimode terminals. In: Proceedings of the 2014 International Conference on Computational Intelligence and Communication Networks, Bhopal, India, pp. 295-299

[5] GPP Group 2011 ETSI technical specification - base station (BS) radio transmission and reception. Release 11 . TS 36.104 v11.2.0

[6] Yilmaz O N C, Hämäläinen J and Hämäläinen S 2010 Selfoptimization of remote electrical tilt. In: Proceedings of the 21st Annual IEEE International Symposium on Personal, Indoor and Mobile Radio Communications, Istanbul, Turkey, pp. $1128-1132$

[7] Johnson C 2010 Long term evolution in bullets, 2nd ed. North Charleston, SC, USA: CreateSpace Independent Publishing

[8] Sesia S, Touffik I and Baker M 2011 LTE-The UMTS long term evolution - from theory to practice, $2^{\text {nd }}$ ed., West Sussex, United Kingdom: Wiley Publication

[9] Shah B, Dalwadi G, Shah H and Kothari N 2018 Energyefficient LTE access network for rural and remote areas. Far East J. Electron. Commun. 18: 1073-1097

[10] Shah B, Dalwadi G, Shah H and Kothari N 2018 Powerefficient LTE macro eNodeB: a comprehensive survey. Telecommun. Radio Eng. 77: 1441-1462

[11] Buenestado V, Toril M, Luna-Ramírez S, Ruiz-Avilés J M and Mendo A 2017 Self-tuning of remote electrical tilts based on call traces for coverage and capacity optimization in LTE. IEEE Trans. Veh. Technol. 66: 4315-4326

[12] Drioli L S, Ostergaard A, Paquay M, Saenz E and Pivnenko S 2011 VAlidation STandard antennas: past, present and future. In: Proceedings of the 5th European Conference on Antennas and Propagation (EUCAP), Rome, Italy, pp. 2532-2536
[13] Hau V X and Ivanov V E 2018 Results of the development and experimental studies of the digital magnetic compass system. In: Proceedings of the 2018 Ural Symposium on Biomedical Engineering, Radioelectronics and Information Technology (USBEREIT), Yekaterinburg, Russia, pp. 359-362

[14] Chengping Y, Lincheng S, Dianle Z, Daibing Z and Zhiwei Z 2014 A new calibration method for vision system using differential GPS. In: Proceedings of the 2014 13th International Conference on Control Automation Robotics \& Vision (ICARCV), Singapore, pp. 1514-1517

[15] Sahoo S K and Roy B K 2014 Antenna azimuth position control using quantitative feedback theory (QFT). In: Proceedings of the International Conference on Information Communication and Embedded Systems (ICICES2014), Chennai, India, pp. 1-6

[16] Hapsari W, Umesh A, Iwamura M, Tomala M, Gyula B and Sebire B 2012 Minimization of drive tests solution in 3GPP. IEEE Commun. Mag. 50: 28-36

[17] Akhpashev R V and Drozdova V G 2018 Spatial interpolation of LTE measurements for minimization of drive tests. In: Proceedings of the 2018 19th International Conference of Young Specialists on Micro/Nanotechnologies and Electron Devices (EDM), Erlagol, Russia, pp. 6403-6405

[18] Chernogorov F and Puttonen J 2013 User satisfaction classification for minimization of drive tests QoS verification. In: Proceedings of the 2013 IEEE 24th Annual International Symposium on Personal, Indoor, and Mobile Radio Communications (PIMRC), London, UK, pp. 2165-2169

[19] Berger S, Fehske A, Zanier P, Viering I and Fettweis G 2014 Comparing online and offline SON solutions for concurrent capacity and coverage optimization. In: Proceedings of the 2014 IEEE 80th Vehicular Technology Conference (VTC2014-Fall), Vancouver, Canada, pp. 1-6

[20] Asghar A, Farooq H and Imran A 2018 Concurrent CCO and LB optimization in emerging HetNets: a novel solution and comparative analysis. In: Proceedings of the 2018 IEEE 29th Annual International Symposium on Personal, Indoor and Mobile Radio Communications (PIMRC), Bologna, Italy, pp. 1-6 